\title{
Perioperative Anxiety, Pain, and Salivary Amylase in Patients Undergoing Pancreatic Surgery
}

\author{
Nanae Ikeda ${ }^{1,2}$, Rie Tomizawa ${ }^{3}$, Rika Moriya ${ }^{4}$, Junko Yamanaka ${ }^{5}$, Hiroyoshi Suzuki ${ }^{2}$, Hidetoshi Eguchi ${ }^{2}$, Hiroaki \\ Nagano $^{2} \&$ Koji Umeshita ${ }^{2}$ \\ ${ }^{1}$ School of Nursing, Mukogawa Women's University, Hyogo, Japan \\ ${ }^{2}$ Graduate School of Medicine, Osaka University, Osaka, Japan \\ ${ }^{3}$ Faculty of Nursing, Senri Kinran University, Osaka, Japan \\ ${ }^{4}$ Faculty of Nursing, Setsunan University, Osaka, Japan \\ ${ }^{5}$ Faculty of Nursing and Rehabilitation, Konan Women's University, Hyogo, Japan \\ Correspondence: Nanae Ikeda, School of Nursing, Mukogawa Women's University, 6-46 Ikebirakicho, \\ Nishinomiya, Hyogo, 663-8558, Japan. Tel: 81-798-399-005. E-mail: n_ikeda@mukogawa-u.ac.jp
}

Received: April 20, 2015

Accepted: May 4, 2015

Online Published: May 29, 2015

doi:10.5539/ijps.v7n2p206

URL: http://dx.doi.org/10.5539/ijps.v7n2p206

\begin{abstract}
Although many reports on perioperative anxiety and stress in patients have been published, very few have longitudinally focused on the acute phase immediately after surgery. We aimed to investigate the actual levels of perioperative stress experienced by patients undergoing pancreatic surgery in the acute phase. We conducted a survey of 29 patients undergoing pancreatic surgery. These patients were assessed five times: once preoperatively and on postoperative days 1,3,5, and 7. The measurement indices used were the State-Trait Anxiety Inventory (STAI), the Visual Analog Scale (VAS) for pain, and salivary amylase activity. No significant difference in the STAI scores was observed throughout the survey period. In other words, the patients did suffer persistent, moderate anxiety. The VAS scores clearly peaked on postoperative day 1 and dropped thereafter. Salivary amylase activity peaked on postoperative day 3 and then decreased. On postoperative day 7, the VAS scores of patients who underwent open surgery were significantly higher than those of patients who underwent laparoscopic surgery; the patients undergoing open surgery also complained of prolonged pain. Significantly higher salivary amylase activity on postoperative day 3 indicated that the open surgery patients experienced greater stress levels. The laparoscopic group experienced less stress than the open group. The results of the present evaluation of anxiety, pain, and stress experienced by patients may provide a very good reference for perioperative care in pancreatic surgery.
\end{abstract}

Keywords: anxiety, perioperative, pain, pancreatic surgery, salivary amylase, State-Trait Anxiety Inventory

\section{Introduction}

Anxiety is a form of stress that can affect the sympathetic and parasympathetic nervous systems (Jawaid, Mushtaq, Mukhtar, \& Khan, 2007). Previous studies have long focused on the stress of surgical patients (Corman, Hornick, Kritchman, \& Terestman, 1958; Caumo et al., 2001; Janis, 1958; Johonson \& Carpenter, 1980; Vingerhoets, 1998). Pick, Molloy, Hinds, Pearce, and Salmon (1994) measured the catecholamine levels of patients undergoing coronary artery bypass graft surgery and determined that fatigue at 30 postoperative days was the greatest in patients with highest perioperative noradrenaline levels. Schubart et al. (2010) focused on the quality of life in postoperative patients undergoing abdominal surgery and reported that the social/family well-being was strongly associated with the quality of life. Aybala, Haluk, Ahmet, and Mustafa (2014) examined the relationship between anxiety levels and social background in patients undergoing gallbladder surgery and reported that a low level of education, female gender, and the relationship status influenced the levels of anxiety.

The prevalence of pancreatic cancer, the early detection of which is difficult, is on the rise worldwide (World Cancer Research Fond International, 2015), particularly in Japan (Matsuda et al., 2013). Japan ranks seventh in the world for age-adjusted prevalence of this disease (8.5 in 100,000 in 2012) (World Cancer Research Fund International, 2015) and is ranked relatively high among Asian countries, where the prevalence of pancreatic cancer is generally low. Surgery is the only radical therapy for pancreatic cancer. However, even with surgical 
intervention, the prognosis is not necessarily good with a 3-year survival rate of Stage 1a (UICC-Stage: International Common Classification), which is the earliest stage, of approximately 50\% in Japan (Egawa et al., 2008). Furthermore, those who undergo surgery suffer through a fairly invasive procedure with a considerable number of postoperative complications (Egawa et al., 2008). Despite various reports on the anxiety of patients undergoing surgery, to the best of our knowledge, there are no reports on anxiety of patients undergoing pancreatic surgery, a procedure which places a large burden on the patient. The aim of this study was therefore to longitudinally examine the stress experienced by patients undergoing pancreatic surgery both subjectively and objectively using the State-Trait Anxiety Inventory (STAI), Visual Analog Scale (VAS) for pain, and salivary amylase activity, with a focus on the acute period before the operation to 1 week after the operation.

\section{Method}

\subsection{Participants}

Adult patients aged $\geq 20$ years undergoing pancreaticoduodenectomy or distal pancreatectomy at Osaka University Hospital who consented to participate in this study were chosen as participants. The patients with metastasis to organs other than the pancreas were excluded. The patients with metastasis to organs other than the pancreas were excluded. Those deemed by the attending physician or ward nurse to be incapable of completing a survey because of their postoperative state were excluded.

\subsection{Survey Method}

\subsubsection{Survey Content}

The Japanese version of the new STAI-Y (STAI-JYZ; hereinafter, STAI), an improved version of the STAI developed by Spielberger, was used (Hidano, Fukuhara, Iwawaki, Soga, \& Spielberger, 2009; Spielberger, 1983). The STAI is a metric for anxiety and can measure state and trait anxiety. In this study, state anxiety, which assesses how individuals currently feel (i.e., fear, tension, nervousness, and concern) (Hidano et al., 2009), was measured. The survey comprised a 20 -item questionnaire with a 4-point rating scale that could be answered in approximately five minutes. The total score ranged from 20 to 80 points, with a higher score indicating greater anxiety.

The VAS for pain comprised a linear $100-\mathrm{mm}$ scale with marks at every $10 \mathrm{~mm}$. The left end of the scale indicated "absolutely no pain" and the right end indicated "the strongest pain imaginable." Participants were asked to indicate an " $\mathrm{x}$ " on the scale denoting their current pain. Marking of " $\mathrm{x}$ " further away from the left end of the scale indicated that the patient felt greater levels of pain.

Salivary amylase activity was measured using a salivary amylase activity monitor (NIPRO, Tokyo, Japan), a portable enzyme analyzer developed by Yamaguchi et al. (Shetty, Zigler, Robles, Elashoff, \& Yamaguchi, 2011; Yamaguchi et al., 2006). Saliva was collected by holding a salivary amylase monitor chip beneath the tongue for 20 seconds. The amount of saliva required for this test was $28 \mu \mathrm{L}$. This device allowed amylase activity to be measured at the bedside for approximately 1 minute after saliva collection (Shetty et al., 2011). Salivary amylase activity measurement has been used in studies as a stress marker because it avoids the stress caused by the invasiveness of blood collection. The measured value reportedly rises when a patient experiences stress (Takai et al., 2004; Takeda, Watanabe, Onishi, \& Yamaguchi, 2008; Yamaguchi et al., 2006).

\subsubsection{Survey Period and Schedule}

Table 1. Content of each measurement time

\begin{tabular}{llllll}
\hline & Preoperatively & $\begin{array}{l}\text { Postoperative } \\
\text { day } 1\end{array}$ & $\begin{array}{l}\text { Postoperative } \\
\text { day 3 }\end{array}$ & $\begin{array}{l}\text { Postoperative } \\
\text { day 5 }\end{array}$ & $\begin{array}{l}\text { Postoperative } \\
\text { day 7 }\end{array}$ \\
\hline STAI & $\circ$ & $x^{\mathrm{a}}$ & $\circ$ & $\circ$ & $\circ$ \\
VAS & $\circ$ & $\circ$ & $\circ$ & $\circ$ & $\circ$ \\
Salivary amylase activity & $\circ$ & $\circ$ & $\circ$ & $\circ$ & $\circ$ \\
\hline
\end{tabular}

Note. ${ }^{a}$ The STAI was not administered on postoperative day 1

$\circ$ shows that it is an investigation item performed.

$\times$ shows that it is the investigation item not performed. 
The survey was conducted from October 2012 to March 2014. Each participant was surveyed five times. The first survey was conducted between the time of hospitalization and the day before the surgery. The second, third, fourth, and fifth surveys were conducted at postoperative days $1,3,5$, and 7 , respectively. Table 1 shows the results of the measurements obtained during each survey. The reason for not administering the STAI on postoperative day 1 was to reduce the pressure on the patient of having to complete the inventory in approximately 5 minutes. The surveys were conducted at the same time of the day with each patient (within 2 hours), with consideration of the diurnal variations in the salivary components. Moreover, because of the effect of diet on the secretion of salivary amylase, there were checks to ensure that participants had not ingested anything within 1 hour of the measurement. Because the surveys were conducted from the period before surgery to the postoperative acute phase, during which the physical and mental burden of patients was predictably large, a ward nurse or the attending physician first determined whether the patients were in a state to participate in the survey.

\subsubsection{Analysis Method}

Because 10 of 20 state anxiety items in the STAI were reverse items, the results from all items were calculated after they were incorporated into the score for each survey following the score adjustment. The score of the VAS for pain was calculated as the actual measured value from the left end of the scale to the " $x$ " mark. Because the baseline of salivary amylase activity varied greatly between the participants, the level of salivary amylase activity at the time of each survey was calculated as the ratio of the postoperative amylase activity level to the preoperative amylase activity level in each participant. The three different measurements were statistically processed using one-way analysis of variance with repeated measures. An independent $t$-test was used to compare the laparoscopic surgery group (hereinafter, laparoscopic group) and open surgery group (hereinafter, open group) at the time of each survey. The level of significance was set at $P<0.05$. The analysis software used was SPSS ver.19.0 (IBM Japan, Tokyo, Japan).

\subsubsection{Ethical Considerations}

Participation in the study was voluntary and participants were free to drop out at any time. Participants were given sufficient written and verbal explanations that they would suffer no disadvantages to their subsequent treatment or hospitalization if they did not participate, that their physical condition would be prioritized particularly after surgery, that they would not be forced to take the survey, that the data obtained would be carefully managed to prevent participants from being personally identifiable, and that the data would be disclosed at any time upon request. Written informed consent was obtained from all participants. The Ethical Review Board of Osaka University Hospital approved this study.

\section{Results}

\subsection{Participant Backgrounds}

Twenty-nine participants (16 males and 13 females) were included in the study. The diseases were pancreatic cancer $(n=14)$, pancreatic endocrine tumor $(n=8)$, pancreatic cystic tumor $(n=6)$, and distal bile duct cancer $(n=1)$. Nine participants underwent pancreaticoduodenectomy and 20 underwent distal pancreatectomy (11 cases of open surgery and nine cases of laparoscopic surgery).

\subsection{STAI}

Cronbach's alpha showed that the internal consistency of the inventory was 0.912 , indicating that this inventory was valid for use in this study.

As shown by the mean values in Table 2, there was no significant difference between the results for the surveys conducted at each time point. Furthermore, no significant differences were observed between the laparoscopic and open groups at any survey time point.

Table 2. Changes in the STAI score

\begin{tabular}{|c|c|c|c|c|}
\hline & Preoperatively & Postoperative day 3 & Postoperative day 5 & Postoperative day 7 \\
\hline All patients $\quad(\mathrm{N}=29)$ & $48.7 \pm 9.4$ & $48.6 \pm 8.0$ & $45.2 \pm 10.1$ & $45.6 \pm 10.5$ \\
\hline Laparoscopic group $(\mathrm{N}=9)$ & $50.6 \pm 7.2$ & $47.6 \pm 7.6$ & $45.9 \pm 7.8$ & $43.4 \pm 8.9$ \\
\hline Open surgery group $(\mathrm{N}=20)$ & $47.8 \pm 10.3$ & $49.0 \pm 8.4$ & $45.0 \pm 11.1$ & $46.6 \pm 11.2$ \\
\hline
\end{tabular}


Note. Mean \pm SD. At the four measurement points, there was no significant difference in "All patients", "Laparoscopic group" and "Open group" by one-way analysis of variance with repeated measures. In each measurement time, there was no significant difference between the "Laparoscopic group" and "Open group" (t-test).

\subsection{Visual Analog Scale}

As shown in Figure 1, VAS scores were very high at postoperative day 1 and decreased gradually, thereafter. Significant differences were observed between the preoperative scores and the scores at postoperative days 1, 3, 5 , and 7. Compared with postoperative day 1, significant differences were observed for all scores. A significant difference was also noted between postoperative days 3 and 7. Almost no variation in scores was seen between the laparoscopic and open groups on postoperative day 1. Scores tended to be higher for the open group than for the laparoscopic group in all surveys from postoperative day 3 , and a significant difference was observed in the scores from postoperative day 7 (Table 3).

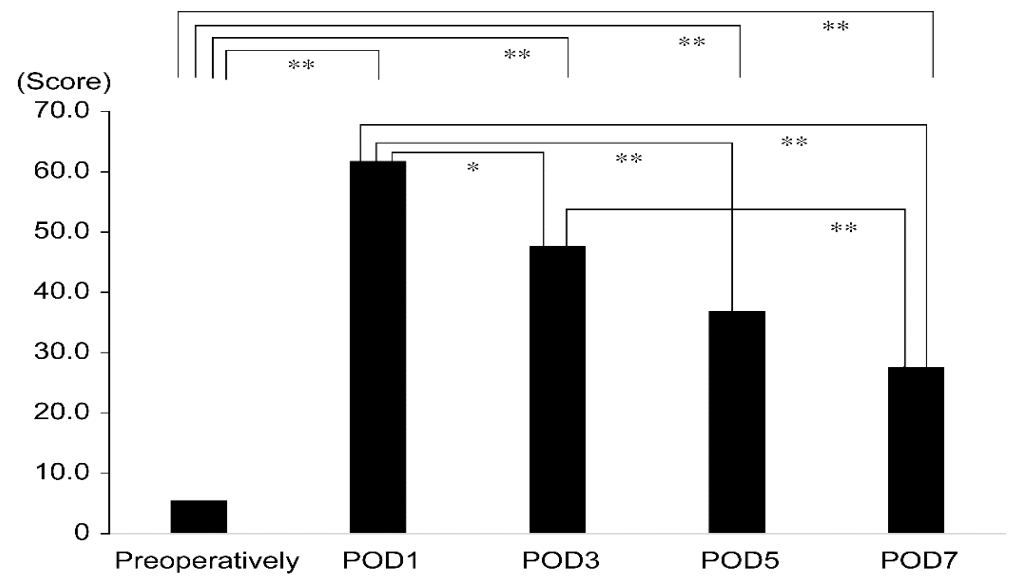

Figure 1. Bar graph of VAS scores of all patients from postoperative days 1 to 7

Note. Each measurement underwent one-way analysis of variance with repeated measures. The level of significance was set at $* p<0.05, * * p<0.01$. In this graph, POD refers to postoperative day. Mean, $(\mathrm{N}=29)$

Table 3. Changes in the VAS score for pain

\begin{tabular}{llllll}
\hline & Preoperatively & $\begin{array}{l}\text { Postoperative } \\
\text { day } 1\end{array}$ & $\begin{array}{l}\text { Postoperative } \\
\text { day } 3\end{array}$ & $\begin{array}{l}\text { Postoperative } \\
\text { day 5 }\end{array}$ & $\begin{array}{l}\text { Postoperative } \\
\text { day } 7\end{array}$ \\
\hline $\begin{array}{l}\text { Laparoscopic group } \\
(\mathrm{N}=9)\end{array}$ & $7.5 \pm 17.53$ & $59.8 \pm 29.6$ & $40.3 \pm 29.9$ & $27.1 \pm 20.5$ & $10.9 \pm 15.1$ \\
Open group (N=20) & $4.8 \pm 12.3$ & $62.8 \pm 25.4$ & $50.8 \pm 24.6$ & $41.1 \pm 25.1$ & $34.3 \pm 24.4$
\end{tabular}

Note. Mean \pm SD. At the point of each postoperative measurement, the values of the Laparoscopic group tended to be lower than those of the Open group. *t-test between the Laparoscopic and Open groups. The level of significance was set at ${ }^{*} p<0.05$

\subsection{Salivary Analyse Activity}

The actual values for preoperative amylase activity of each subject were expressed in ratios equivalent to 1 . As shown in Table 4, the mean values for salivary amylase activity were the highest at postoperative day 3 , although there was no significant difference between the preoperative values. In all the surveys, the open group tended to have higher values than the laparoscopic group (with a significant difference only at postoperative day 3 ). 
Table 4. Changes in salivary amylase activity levels

\begin{tabular}{|c|c|c|c|c|c|}
\hline & Preoperatively & $\begin{array}{l}\text { Postoperative } \\
\text { day } 1\end{array}$ & $\begin{array}{l}\text { Postoperative } \\
\text { day } 3\end{array}$ & $\begin{array}{l}\text { Postoperative } \\
\text { day } 5\end{array}$ & $\begin{array}{l}\text { Postoperative } \\
\text { day } 7\end{array}$ \\
\hline All patients $(\mathrm{N}=29)$ & 1.00 & $2.98 \pm 4.56$ & $4.19 \pm 6.16$ & $2.74 \pm 4.88$ & $2.88 \pm 5.39$ \\
\hline Laparoscopic group $(\mathrm{N}=9)$ & 1.00 & $1.57 \pm 1.4$ & $1.62 \pm 1.68$ & $1.16 \pm 0.72$ & $1.07 \pm 0.75$ \\
\hline Open group $(\mathrm{N}=20)$ & 1.00 & $3.57 \pm 5.30$ & $5.27 \pm 7.04$ & $3.40 \pm 5.71$ & $3.64 \pm 6.30$ \\
\hline
\end{tabular}

Note. Mean \pm SD. The level of salivary amylase activity at the time of each survey was shown as the ratio of the postoperative amylase activity level to the preoperative amylase activity level in each subject. The value was highest on the third day after surgery. At each measurement point, the Laparoscopic group tended to be lower in value than the Open group. ${ }^{*}$ t-test between the laparoscopic group and open group. The level of significance was set at ${ }^{*} p<0.05$.

\section{Discussion}

Till date, many reports of studies on perioperative anxiety and stress in patients have been published both in Japan and abroad (Bodley, Jones, \& Mather, 1974; Corman et al., 1958; Caumo et al., 2001; Edell-Gustafsson \& Hetta, 1999; Janis, 1958; Johonson et al., 1980; Karanci \& Dirik, 2003; Nagasawa, Kitai, \& Nakamura, 2002; Shiromaru et al., 2007; Vingerhoets, 1998). However, most of these studies have compared scores before surgery with those from 1 to 2 weeks after surgery, and very few have focused on the acute phase immediately after surgery longitudinally. Moreover, an investigation using both indices of the subject-object is rare.

The results of the present study showed that STAI scores remained unchanged without any significant difference from the time before surgery to the time after surgery. Patients therefore had a moderate level of anxiety (Edell-Gustafsson \& Hetta, 1999). The preoperative STAI score of 48.7 in the present study was almost the same as that (46.4) obtained by Edell-Gustafsson et al in a study of patients undergoing coronary artery bypass graft surgery (Edell-Gustafsson \& Hetta, 1999). Before surgery, patients experience concern regarding the surgery and anesthesia (Bodley et al., 1974) as well as regarding postoperative treatment and physical pain (Shiromaru et al., 2007). Preoperative explanations regarding the possibility of severe postoperative complications specific to pancreatic surgery and the short life expectancy following successful surgery also may have some effect (Egawa et al., 2008). In this study, there were no major differences between the STAI scores before surgery and seven days after surgery ( 48.7 vs. 45.6$)$. Different results were obtained in the present study compared with those in previous reports, wherein the anxiety level clearly decreased in three to five days or one week after the surgery compared with the levels before surgery (Karanci \& Dirik, 2003; Nagasawa et al., 2002). This may be because of the long fasting period and drain placement period often observed following pancreatic surgery and concerns about serious complications such as anastomotic failure and pancreatic juice leakage during the postoperative period. Concern regarding the fact that their disease was a neoplastic disease also had an effect on patients. Further studies on the specific factors of stress faced by individual patients will be required.

The VAS for pain was primarily used to evaluate postoperative wound pain. A mean preoperative score of 5.5 was obtained. Abdominal pain and lower back pain are known symptoms of pancreatic cancer and these may have had an impact on the scores (Egawa et al., 2008). However, no questions in the present survey were related to the site of the pain. Subjects aged $\geq 65$ years accounted for $58.6 \%$ of all participants; therefore, symptoms such as lower back pain and knee and shoulder joint pain suffered by the elderly may have influenced the findings (Hirase et al., 2014). When dealing with patients, information regarding the cause of each individual patient's pain will need to be broadly collected, keeping in mind the presence of preoperative pain.

The VAS scores at postoperative day 1 were significantly higher in all surveys, revealing that the most pain was felt at this point of time. Wound pain is considered the main cause of postoperative pain and is the most intense at 24-72 hours after surgery, after which it decreases with time (Avidan, Harvey, Ponte, Wendon, \& Ginsburg, 2003). The results of the present study supported this assertion. Through excitement of the sympathetic nervous system, postoperative pain can affect the cardiovascular system, causing elevated blood pressure and tachycardia, and can lead to respiratory complications, muscle weakness, and delay in ambulation (Avidan et al., 2003). Postoperative pain thus needs to be sufficiently controlled on the assumption that the strongest pain is felt one day after surgery. VAS scores dropped significantly three days after surgery, after which the scores remained on a downward trend. However, pain was found to persist even seven days after surgery. At postoperative day 2, the physical pain from the recovery of intestinal peristalsis and increased ambulation were also involved in the 
overall feelings of pain in addition to wound pain (Shiga \& Takeuchi, 2012). Therefore, it needs to be understood that the degree and factors of pain transform following surgery as time passes. Explaining the predicted course of pain to patients in specific numerical values may also aid in supporting them during the acute postoperative phase.

Meanwhile, salivary amylase activity increased from before surgery to after surgery and then decreased after peaking at postoperative day 3. There were no significant differences throughout the survey period. Amylase activity is a real-time biomarker of reactions during stress (Rohleder \& Nater, 2009; Uesato et al., 2010). Changes in amylase activity levels can sometimes effectively indicate the presence of stress in a timely manner as opposed to reflecting past stressful events. Salivary amylase activity reflects both mental and physical stress; therefore, it is difficult to selectively evaluate either type of stress. After surgery, salivary amylase activity may reflect the effects of surgical invasiveness on the body as well as stress that patients are aware of, such as stress from the presence of pain, from limited mobility, and from the discrepancy between the imagined and actual postoperative courses. The results of this study demonstrated that patients experienced greater mental and physical stress three days after surgery than at one day after surgery; these findings may serve as a reference in future perioperative management.

In this study, the results of each measurement were compared between the laparoscopic and open groups. No difference was observed between the two groups throughout the study period for STAI scores, and VAS scores in both groups were almost the same at postoperative day 1 . These results were different than expected. From three to five days after surgery, the laparoscopic group had lower VAS scores than the open group and there was a significant difference at 7 days after surgery, which was as expected. An examination of salivary amylase activity revealed that activity levels remained lower in the laparoscopic group than in the open group throughout the study period. The difference was significant during the peak postoperative day 3 , at which time the level was 1.62 times that of the preoperative level in the laparoscopic group and 5.27 times that of the preoperative level in the open group. Laparoscopic surgery is considered to have some advantages because it is minimally invasive (Iacobone, Citton, \& Nitti, 2012; Liag, Hameed, \& Jayaraman, 2014), and the results of the VAS and salivary amylase activity supported this conclusion.

Our study had several limitations. First, it consisted of a survey of patients undergoing pancreatic surgery performed at a single facility, which limited the utility of the results. Second, this study surveyed only a small sample of patients, and because of this, we were able to demonstrate an overall tendency rather than draw definite conclusions. We intend to perform further investigation including larger number of patients and different types of diseases in the future. Third, only salivary amylase activity was measured because small quantities of saliva were obtained from the postoperative patients. Measurement of other salivary stress markers such as cortisol and secretory IgA would give us more information. The development of a device that can measure multiple biomarkers from small quantities of saliva for these biomarkers could be beneficial.

\section{Conclusion}

The stress experienced by patients undergoing pancreatic surgery was evaluated by measuring the STAI, the VAS for pain, and salivary amylase activity at five points in time before surgery up to seven days after surgery. The results revealed no changes in STAI scores before and after surgery. VAS scores for pain clearly peaked at postoperative day 1 and decreased thereafter. Salivary amylase activity peaked at postoperative day 3 and subsequently decreased. Significant differences between the laparoscopic and open groups were noted at postoperative day 7 for VAS and at postoperative day 3 for salivary amylase activity. These findings suggested that the laparoscopic group experienced less stress than the open group. We were able to determine an overall tendency using concrete numerical values for pain and stress in patients who underwent pancreatic surgery. We believe that these findings could lead to improvement in the active recovery of such patients postoperatively through the medical staff explaining to patients preoperatively that pain and stress are to be expected and by providing physical and moral support.

\section{Acknowledgments}

We would like to express our deepest gratitude to all the patients and their families for their cooperation in this study during a time of great mental and physical stress as well as all the doctors and nurses from the facility where this study was conducted for their kind cooperation. We obtained no funding.

\section{References}

Avidan, M., Harvey, A. M. R., Ponte, J., Wendon, J., \& Ginsburg, R. (2003). Perioperative care, anaesthesia, pain management and intensive care. London: Churchill Livingstone. 
Aybala, A. A., Ulucanlar, H., Ay, A., \& Ozden, M. (2014). Risk factors for perioperative anxiety in laparoscopic surgery. Journal of the Society of Laparoendoscopic Surgeons, 18(3). http://dx.doi.org/10.4293/ JSLS.2014.00159

Bodley, P. O., Jones, H. V. R., \& Mather, M. D. (1974). Preoperation anxiety: A qualitative analysis. Journal of Neurology, Neurosurgery and Psychiatry, 37(2), 230-239. http://dx.doi.org/10.1136/jnnp.37.2.230

Caumo, W. et al. (2001). Risk factor for preoperative anxiety in adults. Acta Anaesthesiologia Scandinavica, 45(3), 298-307. http://dx.doi.org/10.1034/j.1399-6576.2001.045003298.x

Corman, H. H., Hornick, E. J., Kritchman, M., \& Terestman, N. (1958). Emotional reactions of surgical patients to hospitalization, anesthesia and surgery. The American Journal of Surgery, 96(5), 646-653. http://dx.doi.org/10.1016/0002-9610(58)90466-5

Edell-Gustafsson, U. M., \& Hetta, J. E. (1999). Anxiety, depression and sleep in male patients undergoing coronary artery bypass surgery. Scandinavian Journal of Caring Sciences, 13(2), 137-143.

Egawa, S., Toma, H., Ohigashi, H., Okusaka, T., Nakao, A., Hatori, T., \& Tanaka, M. (2008). A digest of the pancreatic cancer registry report 2007. Journal of the Japan Pancreas Society, 23, 105-123. http://dx.doi.org/10.2958/suizo.23.105

Hidano, T., Fukuhara, M., Iwawaki, S., Soga, S., \& Spielberger, C. D. (2009). Manual for the State-Trait Anxiety Inventory (New Edition). Tokyo: Jitsumukyoiku-Shuppan.

Hirase, T., Kataoka, H., Inokuchi, S., Nakano, J., Matsusaka, N., \& Okita, M. (2014). Factors affecting activities of daily living due to musculoskeletal pain in community-dwelling older adults. Journal of Musculoskeletal Pain Research, 6, 99-106.

Iacobone, M., Citton, M., \& Nitti, D. (2012). Laparoscopic distal pancreatectomy: Up-to-date and literature review. World Journal of Gastroenterology, 18(38), 5329-5337. http://dx.doi.org/10.3748/wjg.v18.i38.5329

Janis, I. L. (1958). The Psychoanalytic of Surgery. Psychological Stress: Psychoanalytic and Behavioral Studies of Surgical Patients. New York, NY: Wiley.

Jawaid, M., Mushtaq, A., Mukhtar, S., \& Khan, Z. (2007). Preoperative anxiety before elective surgery. Neurosciences, 12(2), 145-148.

Johonson, M., \& Carpenter, L. (1980). Relationship between pre-operative anxiety and post-operative state. Psychological Medicine, 10, 361-367.

Karanci, A. N., \& Dirik, G. (2003). Predictors of pre- and postoperative anxiety in emergency surgery patients. Journal of Psychosomatic Research, 55(4), 363-369. http://dx.doi.org/10.1016.S0022-3999(02)00631-1

Liag, S., Hameed, U., \& Jayaraman, S. (2014). Laparoscopic pancreatectomy: Indications and outcomes. World Journal of Gastroenterology, 20(39), 14246-14254. http://dx.doi.org/10.3748/wjg.v20.i39.14246

Matsuda, A., Matsuda, T., Shibata, A., Katanoda, K., Sobue, T., Nishimono, H., \& The Japan Cancer Surveillance Group. (2013). Cancer incidence and incidence rates in Japan in 2008: A study of 25 population-based cancer registries for the Monitoring of Cancer Incidence in Japan (MCIJ) project. Japanese Journal of Clinical Oncology, 44, 388-396.

Nagasawa, M., Kitai, M., \& Nakamura, M. (2002). Evaluation of perioperative anxiety in patients undergoing operation using STAI (Japanese Version) Method. Bulletin of Yamanashi Medical University, 19, 97-100.

Pancreatic cancer statistics. (2012). Data on specific cancers, World Cancer Research Fund International. Retrieved May 22, 2015, from http://www.wcrf.org/int/cancer-facts-figures/data-specific-cancers/ pancreatic-cancer-statistics

Pick, B., Molloy, A., Hinds, C., Pearce, S., \& Salmon, P. (1994). Post-operative fatigue following coronary artery bypass surgery: Relationship to emotional state and to the catecholamine response to surgery. Journal of Psychosomatic Research, 38(6), 599-607. http://dx.doi.org/10.1016/0022-3999(94)90057-4

Rohleder, N., \& Nater, U. M. (2009). Determinants of salivary $\alpha$-amylase in humans and methodological considerations. Psychoneuroendocrinology, 34(4), 469-485. http://dx.doi.org/10.1016/j.psyneuen.2008. 12.004

Schubart, J. R., Wise, J., Deshaies, I., Kimchi, E. T., Staveley-O’Carroll, K. F., \& Gusani, N. J. (2010). Quality of life assessment in postoperative patients with upper GI malignancies. Journal of Surgical Research, 163(1), 40-46. http://dx.doi.org/10.1016/j.jss.2010.04.057 
Shetty, V., Zigler, C., Robles, T. F., Elashoff, D., \& Yamaguchi, M. (2011). Developmental validation of a point-of-care, salivary $\alpha$-amylase biosensor. Psychoneuroendocrinology, 36(2), 193-199. http://dx.doi.org/ 10.1016/j.psyneuen.2010.07.008.

Shiga, Y., \& Takeuchi, T. (2012). Knowledge and skills required for postoperative care, Perioperative nursing for adults and the elderly, 2 intraoperative/postoperative biological reactions and acute nursing. Tokyo: Ishiyaku Publishing.

Shiromaru, M., Shimoda, M., Kubota, M., Yamaguchi, T., Miyasaka, M., TsuTsumi, C., \& Ito, T. (2007). Anxiety before and after surgery for patients who undergo abdominal surgery and specified structure of worries. Journal of the Showa Medical Association, 67, 435-443. http://dx.doi.org/10.14930/ jsma1939.67.435

Spielberger, C. D. (1983). Manual for the State-Trait Anxiety Inventory: STAI (Form Y). Consulting Press, Palo Alto.

Takai, N., Yamaguchi, M., Aragaki, T., Eto, K., Uchihashi, K., \& Nishikawa, Y. (2004). Effect of psychological stress on the salivary cortisol and amylase levels in healthy young adults. Archives of Oral Biology, 49(12), 963-968. http://dx.doi.org/ 10.1016/j.archoralbio.2004.06.007

Takeda, K., Watanabe, M., Onishi, M., \& Yamaguchi, M. (2008). Correlation of salivary amylase activity with eustress in patients with severe motor and intellectual disabilities. The Japanese Association of Special Education, 45, 447-457.

Uesato, M., Nabeya, Y., Akai, T., Inoue, M., Watanabe, Y., Kawahira, H., \& Matsubara, H. (2010). Salivary amylase activity is useful for assessing perioperative stress in response to pain in patients undergoing endoscopic submucosal dissection of gastric tumors under deep sedation. Gastric Cancer, 13(2), 84-89. http://dx.doi.org/10.1007/s10120-009-0541-8

Vingerhoets, G. (1998). Perioperative anxiety and depression in open-heart surgery. Psychosomatics, 39(1), 30-37.

Yamaguchi, M. et al. (2006). Hand-held monitor of sympathetic nervous system using salivary amylase activity and its validation by driver fatigue assessment. Biosensors and Bioelectronics, 21(7), 1007-1014. http://dx.doi.org/10.1016/j.bios.2005.03.014

\section{Copyrights}

Copyright for this article is retained by the authors, with first publication rights granted to the journal.

This is an open-access article distributed under the terms and conditions of the Creative Commons Attribution license (http://creativecommons.org/licenses/by/3.0/). 